EPJ Web of Conferences 38, 05001 (2012)

DOI: $10.1051 /$ epjconf/20123805001

(C) Owned by the authors, published by EDP Sciences, 2012

\title{
Theoretical description of nuclear masses
}

\author{
Yuri A. Litvinov ${ }^{1}$ and Adam Sobiczewski ${ }^{1,2}$ \\ 1 GSI-Helmholtzzentrum für Schwerionenforschung GmbH, 64291 Darmstadt, Germany \\ 2 National Centre for Nuclear Research, Hoża 69, 00-681 Warsaw, Poland
}

\begin{abstract}
Ten different theoretical models are tested for their accuracy in description of nuclear masses. Recent experimental masses of 2011 are used for the tests. The accuracy is studied in three regions of the nuclear chart: global (all nuclei with $Z, N \geq 8$ ), heavy $(Z \geq 82, N \geq 126)$ and the heaviest ones $(Z>100)$. It is found that the accuracy of a given model strongly depends on the region of nuclei in which it is used.
\end{abstract}

\section{Introduction}

There is a continuing progress in the measurement and evaluation of nuclear masses. Between the previous evaluation [1] and the recent one [2], masses of more than 140 nuclei have been measured, in particular of more than 30 heavy (heavier than ${ }^{208} \mathrm{~Pb}$ ) nuclides. Also the accuracy of measured masses has been improved for many nuclei.

In the parallel progress of theoretical description of the masses, a significant reduction of the discrepancy between the calculated values and the measured ones has been achieved. Particularly large reduction has been obtained in the purely microscopic, self-consistent description. For example, a big reduction has been reached in a long series of the (Skyrme)-Hartree-Fock-Bogoliubov (HFB) models: from $0.764 \mathrm{MeV}$ in the first version of the model HFB1 [3], down to $0.574 \mathrm{MeV}$ in the recent one HFB21 [4]. The rms of the HFB1 was obtained with the measured masses of 1888 nuclei, available at that time, while that of the HFB21 was calculated with 2294 masses known presently [2].

The objective of the present paper is to study the quality of the available models in description of the masses. In particular, it is planned to study the dependence of the accuracy of a given model on the region of nuclei in which it is used. Ten models of different kinds: semi-empirical, macroscopic-microscopic (macro-micro), and purely microscopic, are considered.

Much attention is devoted to heaviest nuclei. The reason for this is to get an orientation which of the models can be used for possibly realistic predictions for the experimental studies of superheavy nuclei (SHN) and superheavy elements (SHE). These, presently intensive studies (e.g. [5-12]), are especially difficult and expensive, due to very small cross sections for the synthesis of SHN.

Some discussion of the description of heavy-nuclei masses by macroscopic-microscopic models has been presented in [13].

\section{Considered nuclear models}

As already stated in the Introduction, ten various models are considered in the study. These are: one semi-empirical
(LMZ) [14], five macroscopic-microscopic, two purely microscopic (self-consistent) and two models of other kind. The macroscopic-microscopic models are: the Finite-Range Droplet Model (FRDM) [15], the Finite-Range Liquid Drop Model (FRLDM) [15], the nuclear Thomas-Fermi (TF) [16], the Warsaw model for Heavy Nuclei (HN) [17] (see also [18]), and the Lublin-Strasbourg (LSD) model [19]. The purely microscopic models are: the HFB21 approach [4], mentioned above, which uses the Skyrme interactions, and the HFB approach exploiting the Gogny forces (GHFB) [20]. Two other models are the following: the model of Duflo and Zuker (DZ) [21] and that of Koura et al. (KTUY) [22]

Two of the models (LMZ and HN) are of a local type, specially adapted to describe heavy nuclei with proton number $Z \geq 82$ and neutron number $N \geq 126$. The others are of a global character describing all nuclei with $Z, N \geq 8$.

\section{Results}

Table 1 shows the accuracy of the description of masses by the considered models. The results are given for three regions of nuclei: global (all nuclei with $Z, N \geq 8$ ), heavy $(Z \geq 82, N \geq 126)$, and the heaviest ones $(Z>100)$. Experimental masses are taken from [2]. For each model and each region, the number of nuclei taken in the calculation, $N_{\text {nucl }}$, rms of the discrepancies, Rms, average value of the discrepancies, $\bar{\delta}$, and maximum of the absolute values of the discrepancies, $\max |\delta|$, are given.

The dependence of the rms on the model and on the region of considered nuclei is better seen when presented in a graphical form. This is done in figures 1,2 , and 3.

One can see in figure 1 that the models may be separated into three groups. DZ has the lowest rms. LSD, FRDM,TF, and HFB21 give medium and similar to each other values, while FRLDM, GHFB and KTUY have the largest rms.

When one goes to the heavy nuclei (figure 2), the picture changes quite much. The rms of LSD goes down, close to that of DZ, while the rms of GHFB and KTUY go up to about $1 \mathrm{MeV}$ or even more. The accuracy of LMZ and HN is rather high, as could be expected for these local models, specially adapted for heavy nuclei. Finally, for the heaviest nuclei (figure 3), the picture is very different from the

This is an Open Access article distributed under the terms of the Creative Commons Attribution License 2.0, which permits unrestricted use, distribution, and reproduction in any medium, provided the original work is properly cited. 
Table 1. The Rms, average discrepancy, $\bar{\delta}$, and maximum of the absolute values of the discrepancies, Max $|\delta|$ (all given in MeV), calculated for all $(Z, N \geq 8)$, heavy $(Z \geq 82, N \geq 126)$, and heaviest $(Z>100)$ nuclei, with the use of the specified models. The year of publication of each model and the number of nuclei with measured mass in each of the considered regions, $N_{\text {nucl }}$, are also indicated.

\begin{tabular}{|l|rrrrrrrrrr|}
\hline Model & LMZ & HN & LSD & FRDM & TF & FRLDM & HFB21 & GHFB & DZ & KTUY \\
& $(2000)$ & $(2001)$ & $(2003)$ & $(1995)$ & $(1996)$ & $(1995)$ & $(2010)$ & $(2009)$ & $(1995)$ & $(2005)$ \\
\hline $\mathrm{N}_{\text {nucl }}(Z \geq 8)$ & - & - & 2267 & 2294 & 2293 & 2294 & 2294 & 2294 & 2294 & 2294 \\
$\mathrm{Rms}$ & - & - & 0.600 & 0.645 & 0.629 & 0.768 & 0.574 & 0.784 & 0.374 & 0.690 \\
$\bar{\delta}$ & - & - & -0.028 & -0.062 & 0.027 & 0.057 & 0.031 & -0.108 & -0.029 & -0.048 \\
$\operatorname{Max}|\delta|$ & - & - & 4.34 & 3.64 & 4.61 & 4.17 & 3.20 & 3.23 & 3.01 & 2.63 \\
\hline $\mathrm{N}_{\text {nucl }}(Z \geq 82)$ & 297 & 297 & 289 & 297 & 296 & 297 & 297 & 297 & 297 & 297 \\
$\mathrm{Rms}$ & 0.202 & 0.358 & 0.352 & 0.455 & 0.476 & 0.731 & 0.484 & 1.057 & 0.333 & 0.986 \\
$\bar{\delta}$ & 0.028 & -0.133 & 0.163 & 0.131 & 0.340 & 0.562 & 0.132 & -0.118 & -0.011 & -0.307 \\
$\operatorname{Max}|\delta|$ & 1.12 & 1.13 & 1.43 & 1.95 & 1.75 & 1.92 & 1.33 & 3.23 & 3.01 & 2.38 \\
\hline $\mathrm{N}_{\text {nucl }}(Z>100)$ & 24 & 24 & 23 & 24 & 23 & 24 & 24 & 24 & 24 & 24 \\
$\mathrm{Rms}$ & 0.215 & 0.147 & 0.295 & 0.676 & 0.484 & 1.251 & 0.314 & 1.172 & 0.761 & 1.326 \\
$\bar{\delta}$ & -0.140 & -0.063 & -0.157 & -0.525 & 0.457 & 1.218 & -0.095 & 1.046 & -0.151 & 1.303 \\
$\operatorname{Max}|\delta|$ & 0.69 & 0.39 & 1.09 & 1.95 & 0.82 & 1.92 & 0.92 & 1.70 & 3.01 & 1.93 \\
\hline
\end{tabular}

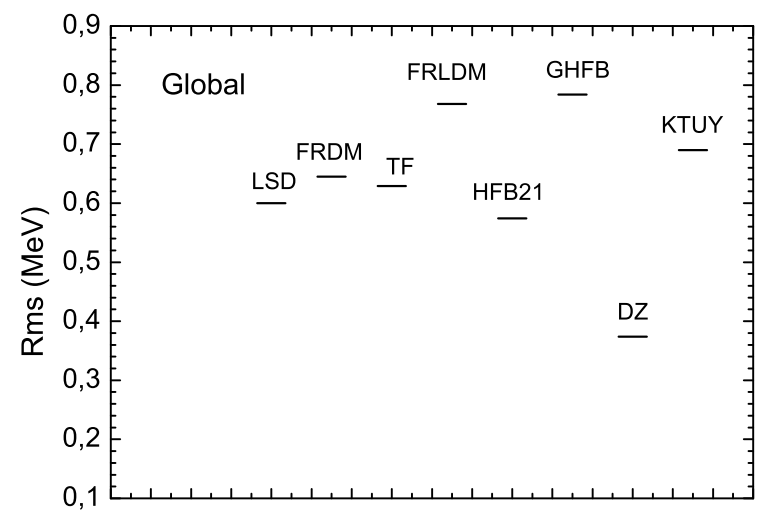

Fig. 1. Rms values of the discrepancies between calculated within 8 global models (see text for the notation of the models) for the whole (global: $Z, N \geq 8$ ) region of nuclei and the experimental values.

previous ones. The rms of $\mathrm{HN}$ is especially low, about 0.15 $\mathrm{MeV}$. Generally good DZ model does not appear to be very successful for nuclei in this region. The rms of FRLDM, GHFB and KTUY are especially large, much above $1 \mathrm{MeV}$.

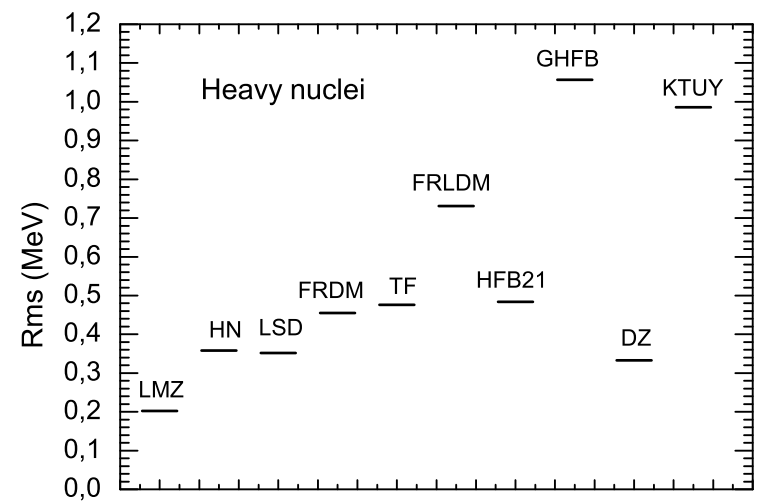

Fig. 2. Same as in figure 1 , but for the heavy-nuclei region $(Z \geq$ $82, N \geq 126$ ). Results for the two local models (LMZ and HN) are also shown.

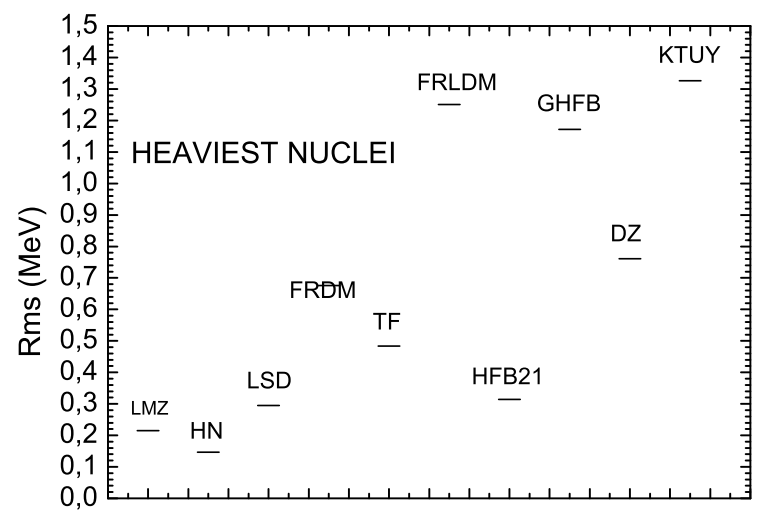

Fig. 3. Same as in figure 2, but for the heaviest region of nuclei $(Z>100)$.

It is worth noting that the self-consistent model HFB21 gives relatively low rms for all three considered regions of nuclei. For the heaviest nuclei, it appears among the models with the smallest rms.

Figure 4 shows a detailed map of the discrepancies obtained for this model in the region of heavy nuclei. The experimental masses of Ref. [2] are taken. The nuclei with

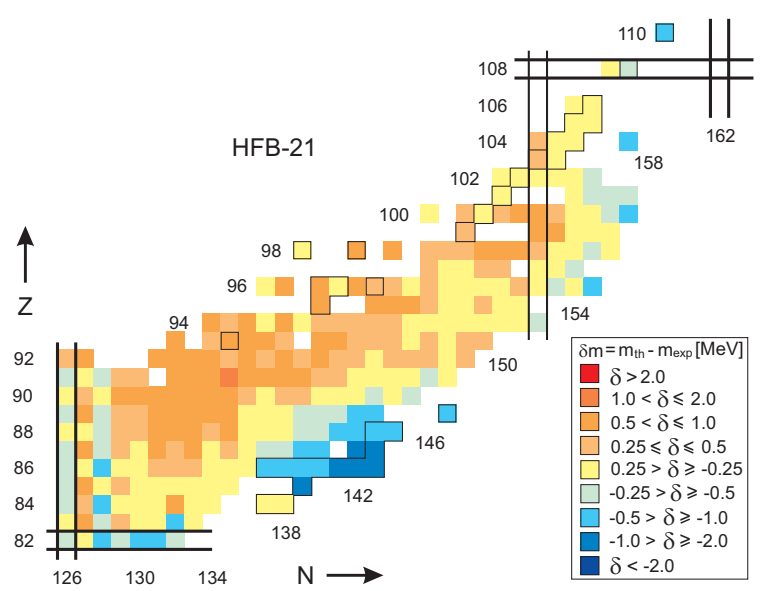

Fig. 4. (Color-online) Detailed map of the discrepacies obtained for the HFB21 model in the heavy-nuclei region. 
masses measured recently (i.e., with masses given in Ref. [2], but absent in Ref. [1]) are put in the thin black frames. Positions of the magic numbers: $Z=82,108$ and $N=126,152$, 162 are indicated by thick black lines. One can see that the discrepancies are below $0.5 \mathrm{MeV}$ (in their absolute values) for most of the nuclei. Only for relatively small number of them, it is larger (up to $1.33 \mathrm{MeV}$ ).

\section{Summary}

Ten different theoretical models are tested for their accuracy in description of nuclear masses. Recent experimental masses of 2011 are used for the tests. The accuracy is studied in three regions of the nuclear chart: global (all nuclei with $Z, N \geq 8)$, heavy $(Z \geq 82, N \geq 126)$ and heaviest ones $(Z>100)$.

The following conclusions may be drawn from the study:

(1) The accuracy of a given model usually strongly depends on the region of nuclei in which it is used. For example, the Duflo-Zuker model (DZ), which has very good average accuracy in both the global and heavy-nuclei regions, is rather poor for description of heaviest nuclei.

(2) The self-consistent model HFB21 gives relatively low rms, lower than that of a number of widely used macroscopic-microscopic models, for all three considered regions of nuclei. This probably happens for the first time, for a purely microscopic model, in the history of description of nuclear masses.

\section{Acknowledgements}

The authors would like to thank Dieter Ackermann, Michael Block, Fritz Bosch, Hans Geissel, Stephane Goriely, Fritz Hessberger, Sigurd Hofmann, and Christoph Scheidenberger for helpful discussions and correspondence. Support by the Helmholtzinstitut Mainz (HIM), the Polish National Centre of Science (within the research project No. N N 202 204938), the European Science Foundation (within the EuroGenesis programme), and the Polish-JINR(Dubna) Cooperation Programme is gratefully acknowledged.

\section{References}

1. G. Audi, A.H. Wapstra, and C. Thibault, Nucl. Phys. A 729, 337 (2003)

2. Georges Audi and Wang Meng, private communication (April 2011)

3. M. Samyn, S. Goriely, P.-H. Heenen, J.M. Pearson, and F. Tondeur, Nucl. Phys. A 700, 142 (2002)

4. S. Goriely, N. Chamel, and J.M. Pearson, Phys. Rev. C 82, 035804 (2010)

5. S. Hofmann, G. Münzenberg, Rev. Mod. Phys. 72, 733 (2000)

6. K.E. Gregorich, et al., Phys. Rev. C 72, 014605 (2005)

7. Yu.Ts. Oganessian, J. Phys. G: Nucl. Part. Phys. 34, R165 (2007)

8. K. Morita, et al., J. Phys. Soc. Japan 76, 045001 (2007)

9. Yu.Ts. Oganessian, et al., Phys. Rev. C 74, 044602 (2006)
10. A. Türler, et al., Eur. Phys. J. A 17, 505 (2003)

11. R. Eichler, et al., Nature (London) 447, 72 (2007)

12. J. Dvorak, et al., Phys. Rev. Lett. 100, 132503 (2008)

13. Yu.A. Litvinov, A. Sobiczewski, A. Parkhomenko, and E.A. Cherepanov, Int. J. Mod. Phys. E 21, 1250038 (2012)

14. S. Liran, A. Marinov, and N. Zeldes, Phys. Rev. C 62, 047301 (2000)

15. P. Möller, J.R. Nix, W.D. Myers, and W.J. Świa̧tecki, At. Data Nucl. Data Tables 59, 185 (1995)

16. W.D. Myers and W.J. Świa̧tecki, Nucl. Phys. A 601, 141 (1996)

17. I. Muntian, Z. Patyk, and A. Sobiczewski, Acta Phys. Pol. B 32, 691 (2001)

18. A. Sobiczewski and K. Pomorski, Prog. Part. Nucl. Phys. 58, 292 (2007)

19. K. Pomorski and J. Dudek, Phys. Rev. C 67, 044316 (2003)

20. S. Goriely, S. Hilaire, M. Girod, and S. Peru, Phys. Rev. Lett. 102, 242501 (2009)

21. J. Duflo and A.P. Zuker, Phys. Rev. C 52, R23 (1995)

22. H. Koura, T. Tachibana, M. Uno, and M. Yamada, Prog. Theor. Phys. 113, 305 (2005) 